\title{
Silent coronary heart disease in patients with type 2 diabetes: application of a screening approach in a follow-up study
}

\author{
Saula Vigili de Kreutzenberg ${ }^{\mathrm{a}, *}$, Anna Solini ${ }^{\mathrm{b}}$, Edoardo Vitolo ${ }^{\mathrm{b}}$, Alessandra Boi ${ }^{\mathrm{c}}$, Simonetta Bacci ${ }^{\mathrm{d}}$, \\ Sara Cocozza ${ }^{\mathrm{e}}$, Rossella Nappo ${ }^{\mathrm{e}}$, Angela Rivellese ${ }^{\mathrm{e}}$, Angelo Avogaro ${ }^{\mathrm{a}}$, Marco Giorgio Baroni ${ }^{\mathrm{f}}$ \\ a Endocrinology and Metabolic Diseases, Department of Medicine - DIMED, University of Padova, Italy \\ b Section of Internal Medicine I, University of Pisa, Italy \\ ${ }^{c}$ Endocrinology and Diabetes, Department of Medical Sciences, University of Cagliari, Italy \\ d Casa Sollievo della Sofferenza Hospital, S. Giovanni Rotondo (FO), Italy \\ e Department of Clinical Medicine and Surgery, Federico II University, Napoli, Italy \\ ${ }^{\mathrm{f}}$ Endocrinology, Department Experimental Medicine, Sapienza University of Rome, and IRCCS Neuromed, Pozzilli (IS), Italy.
}

\section{A R T I C L E I N F O}

\section{Article history:}

Received 19 January 2017

Received in revised form 27 March 2017

Accepted 29 March 2017

Available online $\mathrm{xxxx}$

\section{Keywords:}

Silent coronary heart disease ischemia

Type 2 diabetes

CHD diagnosis

Electrocardiogram

Diabetes complications

Positive predictive value

\begin{abstract}
A B S T R A C T
Aims: The cost-effectiveness of screening for silent coronary heart disease (CHD) in type 2 diabetes (DM2) is still debated.

Methods: We applied a diagnostic algorithm for silent CHD detection, in a cohort of 102 asymptomatic DM2 subjects ( $57 \pm 7$ years), attending 5 Italian outpatient clinics, to verify its predictive value. The risk of silent CHD was calculated considering classical risk factors, and presence of microangiopathy/macroangiopathy. Patients were divided in 3 groups, i.e. group 1: normal ECG and low silent CHD risk; group 2: abnormal ECG, irrespective of silent CHD risk; group 3: high silent CHD risk, irrespective of ECG. To group 2 and 3, a functional test was recommended and performed in $78 \%$ of patients.

Results: Silent CHD prevalence was similar in group 2 and 3 ( 25 vs. 17\% respectively; p $=0.495$ ). However, evaluating the entire cohort, a significant higher prevalence of silent CHD was observed in subjects with abnormal vs. normal ECG ( 23 vs. $4 \%$; $\mathrm{P}=0.004$ ), but not in subjects with high vs. low pre-test silent CHD risk (14 vs. $9 \%$; $\mathrm{p}=0.472$ ).

Conclusions: An abnormal ECG was a strong, independent predictor of silent CHD (OR 8.9; CI 1.27-62.5; p = 0.028) in DM2. Therefore, a functional stress testing should be considered in DM2 patients with ECG abnormalities.
\end{abstract}

(C) 2017 Elsevier Inc. All rights reserved.

\section{Introduction}

Patients with type 2 diabetes mainly die from ischemic heart disease or ischemic stroke, so their life expectancy is reduced by at least 6 years compared to the non-diabetic counterpart. ${ }^{1}$ Any phenotype of heart disease significantly associate with diabetes, as recently confirmed in a wide population study, by Shah et al. ${ }^{2}$ It is also well documented that coronary heart disease (CHD) in diabetes is more severe, ${ }^{3}$ and more frequent than in non-diabetic subjects: about $75 \%$ of diabetic patients without a diagnosis of CHD show high-grade atherosclerotic coronary lesions, at post-mortem examination. ${ }^{4}$ The prevalence of asymptomatic CHD in this population is round $20 \%$, in most studies, ${ }^{5-9}$ a figure higher than the non-diabetic individuals; therefore in type 2 diabetes the presence of silent myocardial ischemia is highly probable. Asymptomatic

Declaration of conflicting interests: The authors declare no potential conflicts of interest with respect to the research, authorship and/or publication of this article. Funding: None.

* Corresponding author at: Dipartimento di Medicine - DIMED, Via Giustiniani, 2, 35128 Padova, Italy. Tel.: + 39049 8216113; fax: + 390498754179.

E-mail address: saula.dekreutzenberg@unipd.it (S. Vigili de Kreutzenberg). myocardial ischemia predicts cardiovascular events in this population, beyond the routine risk prediction. ${ }^{10}$ For this reason, the cost-effectiveness of a screening strategy to detect silent CHD has been proposed although there is no unanimous consensus. Several guidelines have addressed this issue, ${ }^{11-15}$ yet recommendations are often conflicting, ${ }^{16}$ and a definite proof that screening programs to prevent cardiac events in patients with type 2 diabetes are worthwhile cost-effective is lacking. For this reason the Italian Societies for the Study of Diabetes (Società Italiana di Diabetologia, SID and Associazione Medici Diabetologi, AMD), and several Italian Societies of Cardiology and Atherosclerosis (Associazione Nazionale Medici Cardiologi Ospedalieri, ANMCO, Associazioni Ragionali Cardiologi Ambulatoriali, ARCA, Società Italiana di Cardiologia, SIC, Società Italiana per lo Studio dell'Aterosclerosi, SISA) have jointly addressed this topic, by proposing a flow-chart with the definition of the high risk of silent CHD, along with an instrumental diagnostic approach to detect silent CHD, in asymptomatic diabetic patients. $^{17}$

The main aim of this study was to identify major predictors of silent coronary artery disease in T2DM patients with a very high pre-test risk of silent CHD; other aims were: 1 . to verify the adhesion to this proposed 
screening protocol to detect silent myocardial ischemia in five outpatient clinics; and 2. to evaluate, in subjects screened for silent CHD, the cardiovascular outcomes at 12 and 30 months of follow-up.

\section{Subjects}

Five Italian Diabetes Centers participated in this study, and a total of 102 patients were enrolled. Participating centers were: the out-patient Diabetes Clinics of Cagliari University, Napoli Federico II University, Padova University, Pisa University, and S. Giovanni Rotondo (Foggia) Hospital. Recruitment and baseline clinical evaluations were performed from January 2012 to December 2012. Consecutive patients attending the above-mentioned outpatient clinics, who met the inclusion/exclusion criteria, were enrolled. Inclusion criteria were: age 35-65 years; type 2 diabetes diagnosed by ADA criteria since at least 1 year; absence of typical cardiac symptoms (chest discomfort, dyspnea, angina, etc.), and negative clinical history for cardiac disease; a recent (within 1 month) electrocardiogram (ECG); definition of the risk of silent myocardial ischemia on the basis of the Consensus suggested criteria (Fig. 1); willingness to participate in the study, and to attend periodical follow-up visits in the respective centers; signed informed consent. Exclusion criteria were type 1 diabetes; other major organ diseases or advanced chronic diabetes complications (i.e. chronic kidney disease stage $\geq 3$; severe proliferative retinopathy; peripheral arterial disease LéricheFontaine stage $\geq 3$ ), unwilling to participate. The study was approved by the local Institution and Ethical Committee of the University Hospital of Padova, and the approval was extended to all other centers.

\section{Materials and methods}

At baseline visit, clinical history, ongoing therapy, chronic diabetic complications, and blood pressure measurements were recorded in each subject. Main anthropometric (weight, height, BMI, waist circumference), and blood pressure measurements in sitting position were obtained after an overnight fasting, and a blood sample was collected to measure clinical chemistry parameters (glucose, glycated hemoglobin, total cholesterol, HDL cholesterol, triglycerides, AST, ALT, creatinine) by standard methods, in each center. LDL-cholesterol (Friedewald formula) and CKD-EPI GFR were also calculated. A urine spot sample was obtained to measure urinary albumin excretion (as albumin/creatinine ratio).

The enrolled subjects were then subdivided in the 3 following groups (Fig. 2): group 1, patients with a normal ECG report, but without a high probability of silent CHD. An ECG was considered "normal" even with an incomplete right bundle branch block, or with isolated 1st degree atrioventricular block; all other ECG interpretations were considered as "abnormal"; group 2, patients with an abnormal ECG, without a high probability of silent CHD; group 3, patients with high silent CHD risk, independent from ECG abnormalities. Patients included in groups 2 and 3 were then suggested to undergo a functional cardiac diagnostic test, in accordance with the Consensus algorithm (Fig. 2), taking into account patient's preference/contraindications. An ECG was repeated to patients allocated in group 1, as suggested, before the follow-up visit. Ongoing pharmacological therapy was possibly modified, during functional cardiac tests, in accordance with the good clinical practice recommendations.

A first follow-up visit was programmed after 12 months. During this visit, the diabetologist had: 1 . to verify whether the suggested diagnostic algorithm was fulfilled; 2 . to register the diagnostic test results; and 3. to collect the main cardiovascular clinical outcomes (acute myocardial infarction, AMI, transient ischemic attack, TIA, stroke, hospitalization for heart failure, peripheral by-pass or amputation). The anthropometric and biochemical parameters were repeated as outlined in baseline (Fig. 2).

A second follow-up visit was programmed after 36 months, for anthropometric and biochemical parameters, and cardiovascular events collection. Vital status and main cardiovascular event (MACE) occurrence were also investigated afterwards.

All the visits and diagnostic tests were performed in an outpatient regimen.

\subsection{Statistical analysis}

Continuous variables are expressed as mean $\pm \mathrm{SD}$, if normally distributed or median (interquartile range), if non-normally distributed, and categorical variables as percentage. Comparison between two or

\section{To meet criteria of at least one of the three boxes gives an high risk of silent coronary heart disease (CHD)}

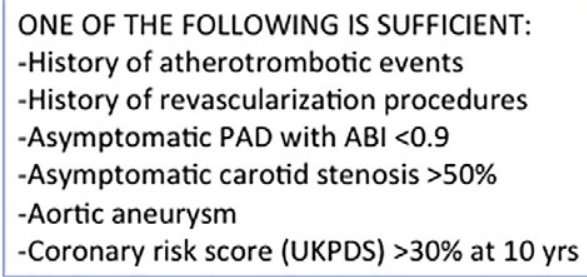

CORONARY RISK SCORE (UKPDS) > 20\% AT 10 yrS + AT LEAST 1 OF THE FOLLOWING:

-Plaque $>20 \%$ stenosis, in any district

-Cardiac autonomic neuropathy

-Erectile dysfunction

$-C A D$ in 1 degree relatives aged $<55$ yrs for women, and $<65$ yrs for male

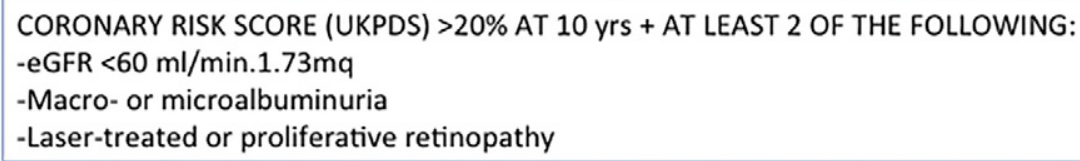

Fig. 1. Definition of the risk of silent myocardial ischemia, as suggested by the Italian Intersociety Consensus. 


\section{BASELINE VISIT}

Demographic, clinical, and biohumoral parameters recording, and group allocation, depending on ECG report and silent CHD risk, to one of the following 3 groups:

\begin{tabular}{|c|c|c|c|c|}
\hline $\begin{array}{l}\text { GROUP 1: } \\
\text { Normal ECG and } \\
\text { low silent CHD risk }\end{array}$ & or & $\begin{array}{c}\text { GROUP 2: } \\
\text { Abnormal ECG } \\
+/- \text { high silent CHD risk }\end{array}$ & or & $\begin{array}{l}\text { GROUP 3: } \\
\text { High silent CHD risk } \\
+/- \text { abnormal ECG }\end{array}$ \\
\hline
\end{tabular}

Recommendation to perform a cardiac test, depending on group allocation:

-GROUP 1: repeat ECG (annually)

-GROUP 2 and GROUP 3: functional cardiac test (stress ECG; if unfeasable perform

SPECT or echocardiogram with pharmacological stress

\section{MONTH FOLLOW-UP VISIT}

-Adherence to suggested diagnostic test evaluation
-Test result recording
-Anthropometric and biohumoral parameters
-CVD events
36 MONTH FOLLOW-UP VISIT
-Anthropometric and biohumoral parameters
-CVD events

Fig. 2. Study protocol.

more groups was performed with the Student's t test or ANOVA, respectively, for continuous variables with normal distribution, and with Wilcoxon or Kruskal-Wallis test, respectively, for variables with non-normal distribution, and with the chi-square test for categorical data. The least significance difference (LSD) post-hoc test was applied. A multiple logistic regression analysis was applied to identify the variables independently associated with CHD.

Statistical significance was accepted at $\mathrm{p}<0.05$, and SPSS ver. 23 was used.

\section{Results}

\subsection{Baseline study}

Main demographic, anamnestic and clinical characteristics of the whole study cohort and comparisons among the 3 predefined groups, at baseline visit, are summarized in Table 1. A different sex distribution was present, among groups; glycated hemoglobin values increased progressively and significantly from group 1 to 3 , while HDL cholesterol concentrations progressively lowered; similarly, the prevalence of arterial hypertension, erectile dysfunction, symptomatic peripheral artery disease, and diabetic retinopathy significantly differed among groups, being maximal in the high silent CHD probability subjects.

The most frequent recorded ECG abnormalities were specific alterations of repolarization (n 7), and left axial deviation (n 7). In 4 patients a $\mathrm{Q}$ wave was diagnosed, in 4 patients a bundle branch blocks, and in 3 patients negative $T$ waves were found.

\subsection{Follow-up at 12 months}

At first follow-up visit ( $12 \pm 0.9$ months) data were collected on protocol adherence, performed diagnostic test, prevalence of silent CHD diagnosis, and main clinical and biohumoral parameters. Eighty-six patients, out of 102 attended the 12-month follow-up visit. The protocol adherence, the frequency and distribution of performed diagnostic tests, and the screening test results are summarized in Table 2 . None of these variable showed statistical differences among groups.

In subjects with positive cardiac functional tests, a coronary angiography (CAG) was performed, to ascertain the presence of significant $(>50 \%)$ coronary stenosis. In all but 1 case, showing on the other hand a positive echo-stress test, silent CHD was confirmed at CAG. Among subjects showing ECGraphic alterations (group 2), 4 male and 1 female had an ascertained diagnosis of silent CHD, while among subjects with a high pre-test risk of silent CHD (group 3), 4 male had an ascertained diagnosis of silent CHD. Of note, in group 2 only 1 patient showed a high pre-test silent CHD risk, and had a negative functional test. No patient of group 1 (normal ECG) showed clinical or ECG/echocardiographic abnormalities at 12-month follow-up visit.

Interestingly, when we compared subjects with normal (n 70) vs. abnormal (n 32) ECG, the prevalence of CHD was significantly higher in patients with ECG alterations (4\% vs. $23 \%$, respectively; $p=0.004$ ), whereas, when we compared subjects with a low pre-test silent CHD risk (n 71) and subjects with a high pre-test risk (n 31), a non-significant difference was observed for the prevalence of CHD within groups ( $9 \% \mathrm{vs}$. $14 \%$, respectively; $\mathrm{p}=0.472$ ).

At this follow-up visit, several anthropometric and biohumoral parameters showed significant improvement, with respect to baseline (Table 3).

Moreover, 2 cardiovascular events were reported: 1 patient of group 1 reported a transitory ischemic attack, and 1 patient of group 2 an episode of heart failure.

\subsection{Diagnosis of silent CHD in patients undergone a functional cardiac test}

When we restricted the analysis to subjects undergoing a functional cardiac screening test (i.e. group 2 and 3; n 44), among patients with normal (n 16) vs. abnormal (n 28) ECG, the diagnosis of silent CHD was confirmed in 2 patients (12.5\%) of the former group, but in 7 patients 
Table 1

Studied variables in the whole cohort, and in predefined subgroups, and comparisons among the 3 subgroups.

\begin{tabular}{|c|c|c|c|c|c|}
\hline Variable & All (n 102) & Group 1 (n 49) & Group 2 (n 23) & Group 3 (n 30) & $\mathrm{p}$ \\
\hline Age (years) & $57 \pm 7$ & $56 \pm 7$ & $58 \pm 7$ & $58 \pm 6$ & 0.402 \\
\hline Gender (M/F) & $55 / 47$ & $22 / 27$ & $9 / 14$ & $24 / 6^{* *}, \#$ & 0.002 \\
\hline BMI $\left(\mathrm{kg} / \mathrm{m}^{2}\right)$ & $33 \pm 8$ & $34 \pm 10$ & $32 \pm 6$ & $33 \pm 7$ & 0.848 \\
\hline Waist $(\mathrm{cm})$ & $111 \pm 17$ & $113 \pm 21$ & $108 \pm 16$ & $109 \pm 12$ & 0.637 \\
\hline UKPDS CHD risk & $17 \pm 10$ & $12 \pm 6$ & $15 \pm 7$ & $27 \pm 10^{* *}, \#$ & $<0.001$ \\
\hline UKPDS fatal CHD risk & $10 \pm 7$ & $7 \pm 5$ & $9 \pm 5$ & $17 \pm 7^{* *}, \#$ & $<0.001$ \\
\hline UKPDS stroke risk & $7 \pm 7$ & $6 \pm 4$ & $6 \pm 4$ & $10 \pm 10^{* *}, \#$ & 0.005 \\
\hline UKPDS fatal stroke risk & $1 \pm 1$ & $0.8 \pm 0.5$ & $0.9 \pm 0.6$ & $1.8 \pm 1.6^{* *}, \#$ & $<0.001$ \\
\hline DM duration (years) & $7 \pm 6$ & $6 \pm 6$ & $7 \pm 6$ & $8 \pm 6$ & 0.717 \\
\hline $\mathrm{SBP}(\mathrm{mmHg})$ & $138 \pm 16$ & $134 \pm 12$ & $137 \pm 15$ & $147 \pm 18^{* *}, \#$ & 0.001 \\
\hline $\mathrm{DBP}(\mathrm{mmHg})$ & $82 \pm 9$ & $82 \pm 8$ & $80 \pm 11$ & $84 \pm 10$ & 0.183 \\
\hline Heart rate (bpm) & $78 \pm 9$ & $77 \pm 8$ & $77 \pm 12$ & $80 \pm 8$ & 0.268 \\
\hline Glucose (mg/dl) & $148 \pm 46$ & $137 \pm 37$ & $150 \pm 59$ & $165 \pm 16^{* * *}$ & 0.032 \\
\hline Glycated haem. (\%) & $7.7 \pm 1.7$ & $7.2 \pm 1.5$ & $7.5 \pm 1.9$ & $8.6 \pm 1.4^{* *}, \S$ & 0.001 \\
\hline Glycated haem. (Mm) & $60 \pm 13$ & $55 \pm 11$ & $58 \pm 15$ & $70 \pm 11^{* *}, \S$ & 0.001 \\
\hline AST $(\mathrm{IU} / \mathrm{L})$ & $24 \pm 11$ & $23 \pm 9$ & $23 \pm 10$ & $27 \pm 15$ & 0.322 \\
\hline $\operatorname{ALT}(\mathrm{IU} / \mathrm{L})$ & $29 \pm 20$ & $27 \pm 22$ & $29 \pm 16$ & $30 \pm 21$ & 0.858 \\
\hline Creatinine $(\mathrm{mg} / \mathrm{dl})$ & $0.92 \pm 0.30$ & $0.89 \pm 0.27$ & $0.88 \pm 0.27$ & $1.00 \pm 0.35$ & 0.180 \\
\hline eGFR $(\mathrm{ml} / \mathrm{min} .1 .73 \mathrm{mq})^{*}$ & $82 \pm 20$ & $83 \pm 19$ & $82 \pm 20$ & $81 \pm 23$ & 0.854 \\
\hline AER (median, interquartile range) (mcg/min) & $10.00(4.08-64.60)$ & $7.50(3.50-15.15)$ & $27.75(7.70-92.00)$ & $12.00(2.55-103.95)$ & 0.130 \\
\hline Tot. cholesterol (mg/dl) & $197 \pm 37$ & $194 \pm 34$ & $203 \pm 38$ & $197 \pm 40$ & 0.365 \\
\hline HDL cholesterol (mg/dl) & $48 \pm 14$ & $51 \pm 14$ & $49 \pm 14$ & $41 \pm 12^{* *}, \S$ & 0.003 \\
\hline LDL cholesterol (mg/dl) & $119 \pm 33$ & $117 \pm 31$ & $116 \pm 34$ & $119 \pm 33$ & 0.453 \\
\hline Triglycerides (mg/dl) & $153 \pm 103$ & $135 \pm 72$ & $158 \pm 110$ & $179 \pm 133$ & 0.188 \\
\hline Hypertension (\%) & 80 & 67 & $87^{\S}$ & $97^{* *}$ & 0.004 \\
\hline Dyslipidemia (\%) & 68 & 59 & 82 & 73 & 0.127 \\
\hline Hypotensive drugs (\%) & 75 & 58 & $86^{* *}$ & $93^{* *}$ & 0.001 \\
\hline Hypolipidemic drugs (\%) & 58 & 44 & $74^{* * *}$ & $70^{* * *}$ & 0.016 \\
\hline Antiaggregants (\%) & 29 & 18 & 39 & 40 & 0.063 \\
\hline Diet $(\%)$ & 10 & 11 & 5 & 10 & 0.782 \\
\hline $\mathrm{OAD}(\%)$ & 77 & 85 & 77 & 63 & 0.080 \\
\hline Metformin (\%) & 76 & 83 & 77 & 63 & 0.146 \\
\hline Sulpho. or glinide (\%) & 21 & 24 & 16 & 20 & 0.761 \\
\hline Incretins (\%) & 16 & 18 & 16 & 13 & 0.879 \\
\hline Glitazone (\%) & 5 & 4 & 16 & 0 & 0.053 \\
\hline Insulin (\%) & 31 & 19 & 40 & $43^{* * *}$ & 0.050 \\
\hline $\mathrm{OAD}+$ Insulin (\%) & 17 & 17 & 16 & 17 & 0.993 \\
\hline Retinopathy (\%) & 17 & 6 & $26^{* * *}$ & $27^{* * * *}$ & 0.022 \\
\hline Nephropathy (\%) & 16 & 8 & 22 & 23 & 0.134 \\
\hline Neuropathy (\%) & 13 & 8 & 22 & 13 & 0.278 \\
\hline Claudicatio $(\%)$ & 8 & 2 & $4^{* * *}$ & $21^{* *}$ & 0.009 \\
\hline Erectile dysfunction (\%) & 7 & 0 & 6 & $19^{* *}$ & 0.008 \\
\hline
\end{tabular}

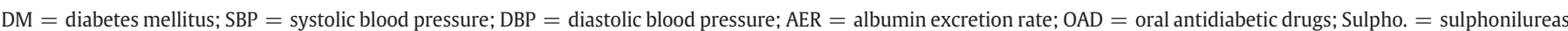

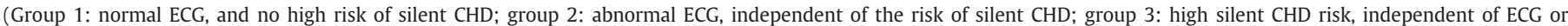
echocardiogram reports.)

Mean \pm SD. ANOVA or Kruskal-Wallis test or chi-squared test p-values and post-hoc least significant differences are shown.

* eGFR was calculated with the CKD-EPI method.

** $\mathrm{p}<0.005$ vs. group 1 .

*** $\mathrm{p}<0.05$ vs. group 1.

\# $\mathrm{p}<0.005$ vs. group 2 .

$\S \mathrm{p}<0.05$ vs. group 2 .

(25\%) of the latter; this comparison however does not reach statistical significance ( $\mathrm{p}=0.322$ ), probably due to the small numbers. Among the 7 patients with abnormal ECG and CHD, 3 showed a low pre-test probability of silent CHD, and 4 a high pre-test probability. On the other hand, both patients with normal ECG and silent CHD showed a high pre-test risk.

When we compared subjects with high silent CHD risk (n 25), calculated as suggested by the Consensus score (Fig. 2), vs. subjects with

Table 2

Adherence to study protocol, performed diagnostic test, and results of silent CHD screening.

\begin{tabular}{|c|c|c|c|c|c|}
\hline & All & Group 1 & Group 2 & Group 3 & $\mathrm{p}$ \\
\hline Subjects $(n)$ & 102 & 49 & 23 & 30 & \\
\hline Protocol adherence/enrolled patients $(\mathrm{y} / \mathrm{n})$ & $80 / 22(78 \%)$ & $36 / 13(73 \%)$ & $20 / 3(87 \%)$ & $24 / 6(80 \%)$ & 0.418 \\
\hline 12-mo FU visit (yes/no) & $86 / 16(84 \%)$ & $36 / 13(73 \%)$ & $22 / 1(96 \%)$ & $28 / 2(93 \%)$ & 0.147 \\
\hline 30-mo FU visit (yes/no) & $66 / 36(65 \%)$ & $32 / 17(65 \%)$ & $10 / 13(43 \%)$ & $22 / 8(73 \%)$ & 0.073 \\
\hline Stress-ECG & $27 / 80$ & 0 & 14 & 13 & - \\
\hline Echo-stress & $9 / 80$ & 0 & 2 & 7 & - \\
\hline MPI & $7 / 80$ & 0 & 4 & 3 & - \\
\hline MSCT & $1 / 80$ & 0 & 0 & 1 & - \\
\hline Positive test in screened patients $(\mathrm{y} / \mathrm{n})$ & $10(14 \%)$ & $0(0 \%)$ & $5 / 15(25 \%)$ & $5 / 19(21 \%)$ & $0.743^{*}$ \\
\hline Ascertained silent CHD by CAG & $9(13 \%)$ & $0(0 \%)$ & $5 / 15(25 \%)$ & $4 / 20(17 \%)$ & $0.495^{*}$ \\
\hline
\end{tabular}

$\mathrm{FU}=$ follow-up. P for ANOVA or ${ }^{*}$ chi-square test between group 2 and 3; MSCT = multi-slice computer tomography. 
Table 3

Comparison of clinical/biohumoral parameters between baseline and 12-month follow-up visits ( $\mathrm{N} 86$ subjects).

\begin{tabular}{lllr}
\hline Variable & Baseline & $\begin{array}{l}12 \text { month } \\
\text { follow-up }\end{array}$ & $\mathrm{p}$ \\
\hline Weight $(\mathrm{kg})$ & $97 \pm 26$ & $92 \pm 23$ & 0.015 \\
BMI $\left(\mathrm{kg} / \mathrm{m}^{2}\right)$ & $34 \pm 10$ & $33 \pm 8$ & 0.025 \\
Waist (cm) & $112 \pm 18$ & $113 \pm 21$ & 0.014 \\
Glucose (mg/dl) & $144 \pm 44$ & $134 \pm 45$ & 0.017 \\
Glycated haem. (\%) & $7.8 \pm 1.8$ & $7.1 \pm 1.4$ & 0.002 \\
Glycated haem. (Mm) & $62 \pm 14$ & $54 \pm 11$ & 0.002 \\
AST (IU/L) & $24 \pm 11$ & $23 \pm 12$ & 0.201 \\
ALT (IU/L) & $30 \pm 21$ & $28 \pm 16$ & 0.427 \\
Creatinine (mg/dl) & $0.92 \pm 0.29$ & $0.89 \pm 0.24$ & 0.035 \\
eGFR (ml/min.1.73mq) & $82 \pm 20$ & $86 \pm 19$ & 0.012 \\
AER (Median, interquartile range) & $10.00(4.08-64.60)$ & $14.00(2.00-108.00)$ & 0.057 \\
$\quad(\mathrm{mcg} / \mathrm{min})$ & & & \\
Total cholesterol (mg/dl) & $203 \pm 36$ & $181 \pm 33$ & $<0.001$ \\
HDL cholesterol (mg/dl) & $47 \pm 15$ & $47 \pm 15$ & 0.925 \\
LDL cholesterol (mg/dl) & $128 \pm 32$ & $106 \pm 25$ & $<0.001$ \\
Triglycerides (mg/dl) & $157 \pm 93$ & $151 \pm 87$ & 0.639 \\
\hline
\end{tabular}

a low pre-test probability ( $\mathrm{n} 19$ ), the prevalence of ascertained silent CHD was not significantly different between groups, though tended to be higher in the low risk group ( $26 \%$ vs. $16 \%$; $p=0.462$ ). Similarly, comparing the patients on the basis of the 10 year UKPDS coronary risk only, i.e. $<20 \%$ ( $n 21$ ) or $>20 \%$ (n 23), the prevalence of silent CHD was alike between groups ( $19 \%$ vs. $22 \%$, respectively; $p=0.825$ ).

These results unravel a stronger association between silent CHD and ECG abnormalities, than between silent CHD and a high disease probability, based on cardiovascular risk factors.

\subsection{Follow-up at 36 months}

The mean duration of this second follow-up visit was $33 \pm 8$ months. Sixty-five percent of the whole group attended the follow-up visit 2 (Table 2). Comparing all studied parameters, between the two follow-up visits (12- and 36-months), a statistically significant difference was observed only for LDL-cholesterol, further reduced at the second follow-up visit $(104 \pm 25$ vs. $97 \pm 28 \mathrm{mg} / \mathrm{dl} ; \mathrm{p}=0.050)$. No other significant changes were observed. At this follow-up visit, 3 more cardiovascular events were reported: 2 acute myocardial infarctions and 1 TIA in patients of group 3; it is of note that all these 3 patients showed an abnormal ECG at baseline.

After a mean period of $33 \pm 8$ months, all patients were alive.

\subsection{Event-free survival at final evaluation}

A further follow-up visit for vital status and main cardiovascular event occurrence was performed at $56 \pm 8$ months. Vital status was known for all the subjects; all were alive, but 1 patient of group 3, who died for pulmonary embolism, after a diagnosis of colon cancer. Data on MACE were available for 91 subjects (89\%), while it was not possible to collect data on MACE for 11 subjects of the initial cohort, at this final

Table 4

Logistic regression analysis to estimate the risk of CHD (dependent variable) associated with major CVD-risk variables.

\begin{tabular}{llll}
\hline Variables & OR & $95 \% \mathrm{CI}$ & $\mathrm{p}$ \\
\hline Age $($ years $)$ & 1.031 & $0.894-1.191$ & 0.672 \\
Gender $(\mathrm{M} / \mathrm{F})$ & 9.541 & $0.476-191.139$ & 0.140 \\
BMI $\left(\mathrm{kg} / \mathrm{m}^{2}\right)$ & 1.147 & $0.985-1.336$ & 0.077 \\
UKPDS CHD risk & 1.019 & $0.907-1.145$ & 0.754 \\
ECG abnormalities & 8.932 & $1.275-62.595$ & 0.028 \\
HbA1c $(\%)$ & 1.094 & $0.543-2.206$ & 0.801 \\
Dyslipidemia & 0.703 & $0.091-5.465$ & 0.737 \\
Hypertension & 0.000 & 0.000 & 0.998 \\
\hline
\end{tabular}

For each odds ratio we estimated a two-tailed p values and 95\% confidence intervals $(\mathrm{CI})$. evaluation. One patient of group 3 experienced a TIA, and 2 patients, one of group 2 and one of group 3, experienced an acute coronary syndrome.

\subsection{Logistic regression analysis of variables associated with $C H D$}

Looking for variables independently associated with the diagnosis of CHD, we analyzed by logistic regression the role of ECG abnormalities, UKPDS CHD risk score, hypertension, HbA1c, and dyslipidemia, together with age, gender and BMI. As shown in Table 4, only ECG abnormalities were independently associated with CHD (OR 8.932, 95\% CI 1.275$62.595, \mathrm{p}<0.028$ ), further confirming its reliability for the screening of silent myocardial ischemia.

\section{Discussion}

The usefulness of screening for asymptomatic CHD in diabetic patients has long been debated, ${ }^{6,11-16}$ but still remains uncertain. In this study we tested the efficacy of the diagnostic algorithm for silent CHD proposed by the Italian Societies for the Study of Diabetes and Cardiology. ${ }^{17}$

We found that in a cohort of unselected asymptomatic type 2 diabetic subjects, the prevalence of silent CHD is $11 \%$; yet this prevalence almost doubled (20.5\%) in patients showing either an abnormal resting ECG or a high pre-test risk of silent CHD. This figure is comparable to that of other studies. ${ }^{5,7,8}$ On the other hand, a low risk of silent CHD, determined by CV risk factors, or a normal ECG are unlikely to be associated with silent CHD.

The high probability of silent CHD, as suggested by our Consensus, is based either on the UKPDS Risk Engine, and/or on the presence of microand microangiopathy complications, which has been shown to be significantly associated with macrovascular disease. ${ }^{18,19}$ Subjects with a high risk score were advised to perform a functional cardiac test, which is also recommended in subjects with an ECG abnormality (apart from the isolated 1st degree atrioventricular block or the incomplete right bundle branch block). We observed a similar prevalence of silent CHD between those with high risk, independent of ECG alterations (group 3), and abnormal ECG, independent of silent CHD risk (group 2). However, when we considered normal vs. abnormal ECG instead of low vs. high pre-test silent CHD risk, the presence of ECG abnormalities seemed to show a better diagnostic performance. To confirm the lesser role of traditional risk factors in identifying diabetic patients at risk of silent CHD, when we compared subjects with vs. without a high UKPDS coronary risk score, the prevalence of ascertained silent CHD between groups was similar.

Conclusively, logistic regression analyses confirmed the independent association of ECG abnormalities with silent CHD.

These findings demonstrate, for the first time in a prospective study, that any ECG alterations should be considered the strongest predictor for silent $\mathrm{CHD}$, while the traditional risk-based approach to identify patients to be screened for silent CHD seems to be of lessened usefulness, as already suggested. ${ }^{20,21}$ Moreover in this study, not only ECG alterations suggestive of myocardial infarction were considered for the diagnosis of silent CHD, as in the population of the UKPDS, ${ }^{5}$ but whatever ECG abnormality, strengthening the importance of this test in evaluating any diabetic individual. The concept that the manifestation of cardiovascular disease, rather than the disease risk definition should be considered in the single patient, has recently been taken into account and exposed in the 2016 ADA Recommendations. While in the 2015 ADA Position Statement, ${ }^{22}$ the screening for CHD was tout-court not recommended, since it failed to improve outcomes, as long as CVD risk factors were treated, in the 2016 recommendations it is suggested "to consider investigations for coronary artery disease in the presence of ... signs or symptoms of associated vascular. disease including carotid bruits, transient ischemic attack, stroke, claudication, or peripheral arterial disease". ${ }^{23}$ This is the first time that overt atherosclerotic disease is suggested as a criterion for silent CHD risk definition, by ADA statements. Also in the Italian intersociety Consensus, the presence of atherosclerosis contributes to the risk definition of silent $\mathrm{CHD}$, but 
compared to this parameter, the presence of specific cardiac alterations, documented at the ECG recording, shows a more important predictive value. Therefore, one relevant observation that we can draw from our results is the importance of performing a standard rest ECG, in all type 2 diabetic patients, every year, as recommended by the Italian Standards of Care for Diabetes. ${ }^{24}$

We found an adherence to the suggested diagnostic protocol in $78 \%$ of patients, which is not satisfactory, given that the patients were managed in specialized centers, and informed about their CHD risk. However, another important result of our study is that main metabolic parameters improved significantly at 1-year follow-up visit, in the whole group, although no particular intervention on lifestyle or drug therapy modifications was given. This observation may suggest that the perception of a closer and wider medical supervision positively affects patient behavior, and confirms that better quality of care is guaranteed in dedicated disease centers. ${ }^{25-27}$

Finally, we observed too few cardiovascular events to draw any conclusion about it, being their incidence of about $2 \%$ per year, in a follow-up of 56 months. This result is comparable to that observed in the subgroup of the DIAD study cohort defined at high-risk by the UKPDS risk engine (about $2 \%$ ).$^{28}$ However, all but one were observed in patients with ECG abnormalities, and only 1 event happened in a low risk subjects with normal ECG.

This study has some limitations, mainly residing in the relatively small number of participants and in the incomplete fulfillment of the diagnostic protocol that may reduce the strength of our results; on the other hand the statistical significance obtained in this small cohort reveals the robustness of the findings.

In conclusion, the presence of whatever ECG abnormality, and not only of Q waves or typical ischemic alterations, can be considered a reliable marker of silent myocardial ischemia, in type 2 diabetic subjects.

\section{Acknowledgements}

None.

\section{References}

1. Emerging Risk Factors CollaborationSeshasai SR, Kaptoge S, Thompson A, Di Angelantonio E, Gao P, Sarwar N, et al. Diabetes mellitus, fasting glucose, and risk of cause-specific death. N Engl J Med. 2011;364:829-41.

2. Shah AD, Langenberg C, Rapsomaniki E, Denaxas S, Pujades-Rodriguez M, Gale CP, et al. Type 2 diabetes and incidence of cardiovascular diseases: a cohort study in 1,9 million people. Lancet Diabetes Endocrinol. 2015;3:105-13.

3. Goraya TY, Leibson CL, Palumbo PJ, Weston SA, Killian JM, Pfeifer EA, et al. Coronary atherosclerosis in diabetes mellitus: a population-based autopsy study. J Am Coll Cardiol. 2002;40:946-53

4. Natali A, Vichi S, Landi P, Severi S, L'Abbate A, Ferrannini E. Coronary atherosclerosis in type II diabetes: angiographic findings and clinical outcome. Diabetologia. 2000;43:632-41.

5. Davis TME, Coleman RL, Holman RR, for the UKPDS Group. Prognostic significance of silent myocardial infarction in newly diagnosed type 2 diabetes mellitus. Circulation. 2013;127:980-7.
6. Rajagopalan N, Miller TD, Hodge DO, Frye RL, Gibbons RJ. Identifying high-risk asymptomatic diabetic patients who are candidates for screening stress single-photon emission computed tomography imaging. J Am Coll Cardiol. 2005;45:43e9.

7. Scirica BM. Prevalence, incidence, and implications of silent myocardial infarction in patients with diabetes mellitus. Circulation. 2013;127:965-7.

8. Tsujimoto T, Kajio H, Takahashi Y, Kishimoto M, Noto H, Yamamoto-Honda R, et al Asymptomatic coronary heart disease in patients with type 2 diabetes with vascular complications: a cross-sectional study. BMJ Open. 2011;2, e000139, http: //dx.doi.org/10.1136/bmjopen-2011-000139.

9. Wackers FJT. Asymptomatic patients with diabetes mellitus should be screened for coronary artery disease. J Nucl Cardiol. 2006;13:609e15.

10. Cosson E, Nguyen MT, Chanu B, Banu I, Chiheb S, Balta C, et al. Cardiovascular risk prediction is improved by adding asymptomatic coronary status to routine risk assessment in type 2 diabetic patients. Diabetes Care. 2011;34:2101-7.

11. Bax JJ, Young LH, Frye RL, Bonow RO, Steinberg HO, Barrett EJ. ADA screening for coronary artery disease inpatients with diabetes. Diabetes Care. 2007:30:2729e36.

12. Chow BJ, Larose E, Bilodeau S, Ellins ML, Galiwango P, Kass M, et al. The "what when, where, who and how?" of cardiac computed tomography in 2009: guidelines for the clinician. Can J Cardiol. 2009;25:135-9.

13. Detection, Evaluation, and Treatment of High Blood Cholesterol in Adults (Adult Treatment Panel III). Final report National Cholesterol Education Program National Heart, lung, and blood institute. National Institutes of Health NIH publication no. 02e5215 September 2002. 2002.

14. Fadini GP, de Kreutzenberg SV, Tiengo A, Avogaro A. Why to screen heart disease in diabetes. Atherosclerosis. 2009;204:11-5.

15. Hendel RC, Berman DS, Di Carli MF, Heidenreich PA, Henkin RE, Pellikka PA, et al. ACCF/ASNC/ACR/AHA/ASE/SCCT/SCMR/SNM 2009 appropriate use criteria for cardiac radionuclide imaging: a report of the American College of Cardiology Foundation appropriate use criteria task force, the American Society of Nuclear Cardiology, the American College of Radiology, the American Heart Association, the American Society of Echocardiography, the Society of Cardiovascular Computed Tomography, the Society for Cardiovascular Magnetic Resonance, and the Society of Nuclear Medicine.J Am Coll Cardiol. 2009:53:2201-29.

16. Ferket BS, Genders TS, Colkesen EB, Visser JJ, SpronkS, Steyerberg EW, et al. Systematic review of guidelines on imaging of asymptomatic coronary artery disease. J Am Coll Cardiol. 2011;57:1591-600.

17. Rivellese AA, Piatti PM, Italian Intersociety Consensus Group. Consensus on: Screening and therapy of coronary heart disease in diabetic patients. Nutr Metab Cardiovasc Dis. 2011;21:757-64.

18. Brownlee M. The pathobiology of diabetic complications: a unifying mechanism. Diabetes. 2005;54:1615e25.

19. Juutilainen A, Lehto S, Rönnemaa T, Pyörälà K, Laakso M. Retinopathy predicts cardiovascular mortality in type 2 diabetic men and women. Diabetes Care 2007:30:292-9.

20. Scognamiglio R, Negut C, Ramondo A, Tiengo A, Avogaro A. Detection of coronary artery disease in asymptomatic patients with type 2 diabetes mellitus. J Am Coll Cardiol. 2006;47:65-71.

21. Wackers FJT, Young LH, Inzucchi SE, Chyun DA, Davey JA, Barrett EJ, et al. Detection of ischemia in asymptomatic diabetics investigators. Detection of silent myocardial ischemia in asymptomatic diabetic subjects: the DIAD study. Diabetes Care. 2004;27:1954-61.

22. American Diabetes Association Position Statement. Cardiovascular disease and risk management American Diabetes Association. Diabetes Care. 2015;38:49-57.

23. American Diabetes Association Position Statement. Cardiovascular disease and risk management American Diabetes Association. Diabetes Care. 2016;39:60-71.

24. Standard italiani per la cura del diabete mellito. www.siditalia.it 2016, http:// www.standarditaliani.it/. Accessed 22 Jul 2016.

25. De Berardis G, Pellegrini F, Franciosi M, Belfiglio M, Di Nardo B, Greenfield S, et al. QuED study. Quality of care and outcomes in type 2 diabetic patients: a comparison between general practice and diabetes clinics. Diabetes Care. 2004:27:398-406.

26. Gnavi R, Picariello R, Karaghiosoff L, Costa G, Giorda C. Determinants of quality in diabetes care process: the population based Torino study. Diabetes Care. 2009;32:1986-92.

27. Gruppo di studio ANNALI AMD. A model of continuous monitoring and improvement of the quality of diabetes care. Epidemiol Prev. 2011;35:18-26.

28. Bansal S, Wackers FJ, Inzucchi SE, Chyun DA, DIAD Study Investigators, et al. Five-year outcomes in high-risk participants in the detection of ischemia in asymptomatic diabetics (DIAD) study: a post hoc analysis. Diabetes Care. 2011;34:204-9. 